\title{
Improvement of a Laboratory Course in Network Analysis: Learning to Validate Knowledge in an Experimental Way
}

\author{
H. E. P. Tattje and H. Vos
}

\begin{abstract}
A laboratory course in Network Analysis has been thoroughly revised. Passing rates, averaged over four years, rose from $48 \%$ to $79 \%$. The major goal of the course was to teach students to experiment and investigate in a systematic way, thus validating knowledge by themselves. This paper reports on the changes introduced and the results obtained. Important features are that difficulties that the students meet in measuring or calculation procedures are distinguished from the basic approach of investigation and practiced in separate assignments, and that the necessary information is complete, concise, general and understandable, but not cookbook-like. Help that students needed from the teaching assistants during the labs diminished considerably, leaving assessment of the logbooks as their main task.
\end{abstract}

\section{INTRODUCTION}

$\mathbf{M}$ ORE students learn more in less time with less effort from the teaching assistants. This is the outcome of an educational improvement of a laboratory course in Network Analysis reported in this paper. Laboratories are pedagogically important because they provide a concrete basis for the formal and very abstract concepts used in Electrical Engineering [1]. The motive to start this project was the wish of the dean of education of the department to diminish the cost of the labs. The faculty responsible for the labs was willing to change them because the educational goals were not attained by a substantial number of the students.

The objectives of the laboratory course in Network Analysis are rather high (cf. [2]): students have to apply new concepts about electrical networks, using measurement apparatus in new ways, thereby experimenting systematically. The students have to develop a method to tackle experimental investigation problems. To validate any result they get they have to find one or more independent procedures providing confirmation (within the limits of accuracy and precision of the procedures). These requirements led to low passing rates.

In the past about $48 \%$ of the students passed the course at the first attempt. An additional $13 \%$ performed satisfactory after remedial tasks were fulfilled in the next trimester. These figures show that quite a number of students did not get the idea, viz. how to experiment systematically and independently. Guidance during the lab hours was intense (and costly), but

Manuscript received September 16. 1992: revised July 13, 1994.

The authors are with the Department of Electrical Engineering, University of Twente, P.O. Box 217, 7500 Enschede, The Netherlands. IEEE Log Number 9407861 not effective in this respect. Therefore a change in teaching was proposed, the content remaining unchanged.

The purpose of the educational change could be stated as follows. About half way through the course most students should have reached a sufficiently high level to be able to experiment independently (no cookbook) in a serious way. The required final level of performance should not change. Neither the time devoted by the students nor the teaching assistants' time should increase. A passing rate of at least $75 \%$ should be reached.

The basic approach used in redesigning the course can be stated as follows. The objectives of the course were stated more explicitly, and in consequence a methodical scheme for experimenting was developed. The way students performed in measuring procedures and calculations has been analyzed. Difficulties in applying procedures have been distinguished from difficulties in approach and lack of prior knowledge, and separated in different assignments. The necessary information has been written down explicitly, in a complete but concise and general form. The general educational principles applied are described elsewhere [3].

In our opinion the pedagogical problems met are general, and our analysis and solution can be generalized to other courses. However, circuits labs and educational systems differ from university to university and country to country. In order to be able to relate the pedagogical problem and our solution to other courses we first present an outline of the course and its contents and of our university system [4].

\subsection{Course}

Network Analysis is a first year's course in the Electrical Engineering Department of the University of Twente in The Netherlands. Dutch law does not allow universities to select students; any student who has passed mathematics and physics of secondary school at the highest level should be admitted. The percentage of students finishing the first year of the curriculum within one (physical) year is extremely low (around $20 \%$ ). After the second (physical) year this percentage is about 60 . Thus about $40 \%$ of our students change department, school or university. The triple function of the first year at university is to give students an orientational view of the study they choose, to select those who are suitable for the study and to refer to other possibilities of study for those who are not. Our course has to contribute to these functions. 
The course is given in the second trimester of the first year. During each trimester of 9 weeks the students take 4 or 5 subjects and attend laboratory classes. A trimester is followed by a period of 4 weeks during which students prepare for examinations and take these. Before commencing with Network Analysis, students have attended an introductory laboratory course on Measuring Instruments (multimeter, oscilloscope, function generator, DC- and AC-signals) and Measuring Methods in Physics. They have also attended introductory courses in Semiconductor Electronics, Calculus, Linear Algebra and Digital Techniques. Network Analysis is applied in a course on Electronics in the third trimester.

Every year about 160 students attend the course, which consists of three parts that run in parallel: lecturing, tutoring and labs. The lectures ( 28 hours in all) are presented in the same period of time. Tutoring hours $(18 \mathrm{~h})$ are attended in groups of about 25 students, as are the laboratory classes ( 9 half days of $3 \frac{1}{2} \mathrm{~h}$ each). Students are expected to spend about 55 hours of study at home. The students' results in the labs are graded separately from the theoretical part of Network Analysis [5].

Students work in pairs in order to promote discussion and mutual help. They have to write down all they think, do or omit in logbooks, beside the results they achieve [6]. A separate logbook is required for each part of the course. One teachingassistant is available for eight students. The assistants are instructed not to demonstrate what students should themselves calculate or measure. The three logbooks are assessed by the teaching assistants. When grading the logbooks, the assistants make written comments. Criteria used are (among others): does the student understand what he/she is doing? does he/she tackle the problem in a systematic way? The first logbook is returned to the student the following session in order to provide a fast and effective feedback. The final mark is based mostly on the marks awarded for the last two logbooks.

\subsection{Contents}

The subject of the laboratory course in Network Analysis is input-output (IO) systems in simple electrical networks. The students have to build (simple) electrical circuits and connect measuring apparatus. The circuits usually consist of a resistor, a capacitor and/or an inductance in different combinations. A multimeter, double-beam oscilloscope (grounded input), DC-voltage source and Krohn-Hite signal generator are used in the laboratory. Students have to learn and use practical concepts like ground, node, short, port and all functions of the apparatus. The difference between the working schematic as a representation of the real circuit as it is constructed (with real components and leads), and the formal, theoretical schematic as a representation of an ideal network (containing ideal elements connected by ideal nodes), is stressed.

The course consists of three parts: introductory measurements, time domain and frequency domain. In the first part the use of the measuring apparatus is extended to signals and responses of several forms (sine, square, triangle, etc). All twenty knobs and switches of the oscilloscope and the function generator each, have to be mastered individually. Construction of measuring schematics is learned. Students learn to avoid shorts when using two or three grounded instruments and they observe the influence of different position of leads on results. They learn to measure the product $\mathrm{RC}$ in different ways using AC-methods.

In the second part (time domain) signals and responses are studied as a function of time for simple passive, timeinvariant IO-systems. The impulse response is measured as the limit of the response to increasingly shorter and ever larger square signals. In the laboratory course theoretical concepts that have just been taught have to be applied: step response, impulse response, network properties like linearity and signal properties such as superposition. Students also have to apply formal procedures like solving differential equations and calculating the convolution integral to simple RC and CR IO-systems and square or triangular input signals.

In the third part, the students meet the new, unknown frequency domain. Now IO-systems, signals and responses are studied as a function of frequency, i.e. timeless. The theory involves transfer function, and multiplication of complex functions, applied to RC and RLC networks. Students have to measure and construct Bode plots for some simple circuits, and use them in constructing polar diagrams.

These descriptions of the course and its contents fit both the old and the new situations. In Section II the analysis, the new elements and the new organization of the course are presented compared to the old way of teaching. Section III describes the data collection, and the results: passing rates and performance of the students. In Section IV conclusions and a discussion of the final results are given.

\section{Changes in the Laboratory Course}

A first analysis brought to light that many students did not understand the idea of systematic investigation and that the tasks were too demanding from the beginning on. Learning to experiment, to solve problems with the apparatus and to apply new theory was too much at one time. Using more time or lowering the final requirements was out of the question. Therefore we further analyzed the instructional process.

A number of shortcomings in the information flow to the students were discovered. A lot of information was given orally by the teaching assistants, either before the blackboard or individually. The explanations were too haphazard, however. Students sometimes sidestepped difficulties they were intended to meet in assignments. Assistants sometimes did not realize the difficulties of the students. Assistants were instructed not to demonstrate measurements or calculations which the students have to find out by themselves, but in practice demonstration was necessary to get students working.

Starting from an explicit formulation of the aims of the laboratory course we now present an end-means analysis of the concepts and skills students have to learn. First we discuss the types of tasks we could distinguish. Then we present the order of the tasks as given, comparing the old and the new situation. Third the relation between the written information and personal, oral, information is presented, in the old and the new situation. 


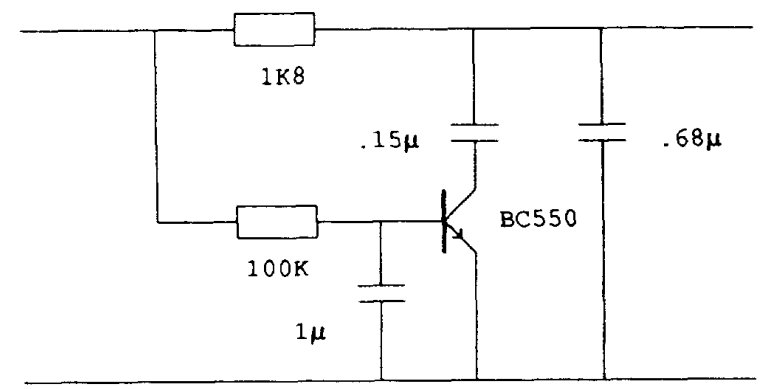

Fig. 1. A seemingly linear circuit. The circuit is made according to the schematic and enclosed in a box (black-box). The measured circuit behavior (cf. Assignment 1) is linear when either harmonics or DC are used, but superposition of a harmonic and DC reveals nonlinearity.

\section{I Types of Tasks}

The most general aim is to learn to investigate and experiment independently. Students should learn how to make sure themselves that their results are correct, instead of asking the teaching assistant. The main notion they have to acquire is that the result of a measurement and that of a calculation always have to fit (within certain limits of accuracy). This basic approach is not at all simple to learn.

A measurement usually involves an--often somewhat distorted and always partial-picture of a signal on an oscilloscope screen. A calculation often results into a formula. These results always differ qualitatively, but they have to be compared. A quantitative correspondence is required. When the results differ quantitatively the students should check their measurement procedure, calculation, circuit, theoretical model, and/or conclusion. Many types of skills are involved, not only thinking skills, but also observational and manipulatory ones. Each type of skill involves many partial skills, like modelling, formula handling, representing results etc.

To help the students a scheme of investigation was designed in which the (partial) skills to be used are summarized (cf. Appendix). According to this scheme students do not get a cookbook for measurements, analysis and conclusions, but they have to construct the cookbook themselves from more general principles and skills. In the past the instructions in the cookbook were not detailed enough, in the new situation it was required that the general directions to construct the cookbook are complete. Special types of assignments are needed to practice such constructions.

Special open-ended tasks were introduced to develop a strategy for selecting and combining these skills, i.e. to develop a strategy for investigation. The characteristics of these openended tasks lead in a natural way to partial skills that are more simple and should be practiced prior to open ended tasks.

In an open-ended assignment neither the quantity or relation to be measured is given, nor the way how that quantity can be determined, nor the criterion that is needed to determine if the task is completed. In open-ended assignments the measuring and calculation methods to be used and the criterion for finishing the investigation all have to be constructed by the students themselves. An example can be found in Assignment 1 .
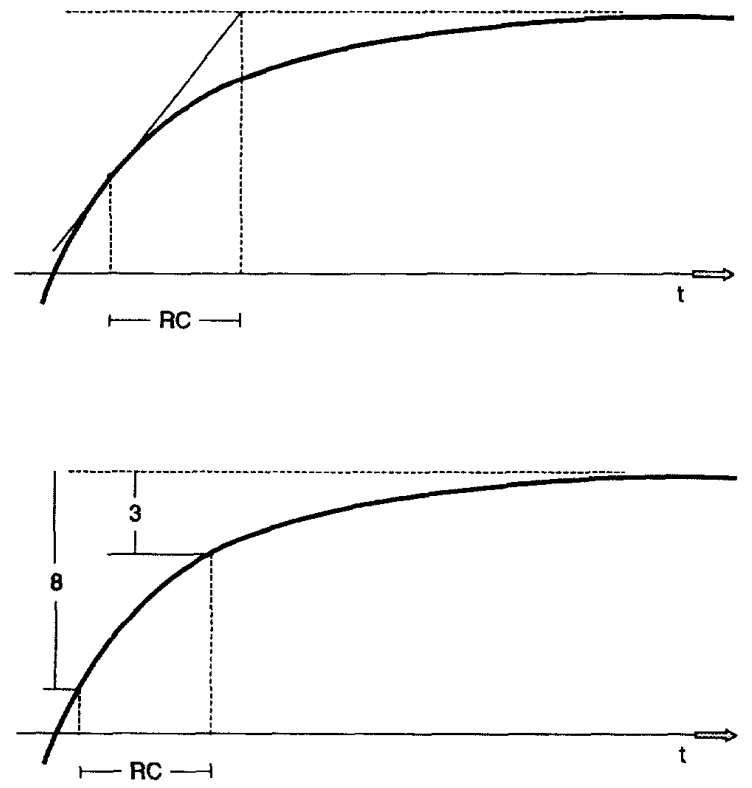

Fig. 2. Methods for the determination of RC. The ratio method uses an approximation of $\mathrm{e}(1 / \mathrm{t} \approx 3 / 8\}$.

Assignment 1. Investigation of Nonlinearity: The assignment reads "Show the nonlinearity of the circuit on your desk using the harmonic signals and the DC-offset of the Krohn-Hite signal generator."

Note. Students are provided with the circuit of Fig. I enclosed in a box. In order to determine the nonlinearity of a circuit one has to choose a method, e.g. an input-output ratio method (harmonics or DC), a phase method or superposition. No explanation of the circuit's behavior is required. Using ratio methods one finds the circuit to be linear.

In order to perform open-ended experiments the students have to be able to choose between appropriate measurements or calculations. Therefore comparative tasks are introduced in which the students have to compare two measurements of a given quantity or relation, or a measurement and a calculation. In comparative tasks the quantity is given, but the methods and criterion still have to be generated (usually the criterion is accuracy). Examples are given in Assignments 2 and 3.

Assignment 2. Comparison of Measuring Methods: The assignment reads: "The product $\mathrm{R}^{*} \mathrm{C}$ is an important quantity of this (resistor-capacitor) circuit. Choose two different methods to measure this quantity. Compare the accuracy of the methods."

Note. The possible methods are in this case the use of square wave or sinusoidal signals. In the first case there are several ways to determine $\mathrm{RC}$ (tangent method, ratio method (see Fig. 2); use of step, square or impulse signals). In the second case it is possible to measure either amplitude or phase differences. Again several procedures are appropriate (for the phase difference three procedures are available: direct, relative and Lissajous).

Assignment 3. Comparison of Measurement and Calculation: The assignment reads: "Calculate and measure the response of 


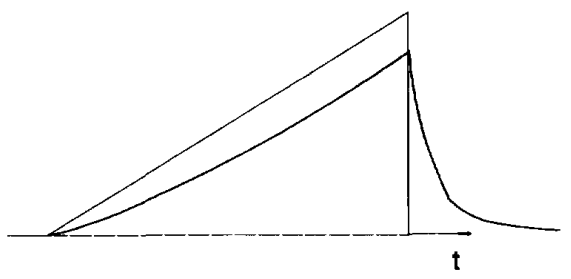

Fig. 3. A triangular signal and the response of an RC circuit to this signal (cf. Assignment 3).

the RC-circuit to a triangular signal with height A (arbitrary) and length $5 * \mathrm{RC}$ (circuit value). Draw the signal and response in one graph" (see Fig. 3).

Before comparative tasks can be fulfilled students have to get preparative tasks to exercise new types of measurements or calculations. Successive tasks are so designed that measurement and calculation procedures have to be adapted to each task, and preferably can not be repeated by plugging different numbers in the same procedure. For instance, in order to be able to calculate the triangular response of Assignment 3 the students first get an assignment to calculate the response to a square wave signal. When they have to measure phase differences at several, deliberately chosen frequencies, they first get an assignment to measure a phase difference at one frequency in three different ways.

Special preparative tasks (introductory tasks) introduce a new concept or a new phenomenon in an experimentally operational way. For instance the concept of step response is introduced as the limit of responses to ever longer square signals. Separate assignments introduce the concept of electrical ground. Students find out, for example, that it is not possible to measure current and voltage in a circuit at the same time, because signal generator and oscilloscope are all grounded.

Homework tasks are intended to study formal information needed later on. This information might be about new concepts or skills, and questions about this information have to be answered to elaborate on the information. The information presented should be sufficient to fulfill the tasks. Not more than reading, understanding and combining the information presented is required.

A special feature thought useful and introduced in some assignments is a confrontation between the thoughts of the students and the observed behavior of real circuits. Such a confrontation forces the student to reflect on his or her own ideas and to discuss the subject with other students. An example is given in Assignment 1. Here most students think of linearity at first as a relation between the amplitudes of input and output signals although both in theory and in mathematics they get the more complete definition including superposition. Using the ratio method they have to conclude the circuit to be linear while they have to find nonlinearity. Thus they are forced to correct their idea about linearity.

Another example is given in Assignment 4. This example concerns the partial functions realized in measuring apparatus. These functions have to be understood and related, in order to

\section{period $0.4 \mathrm{~ms}$}

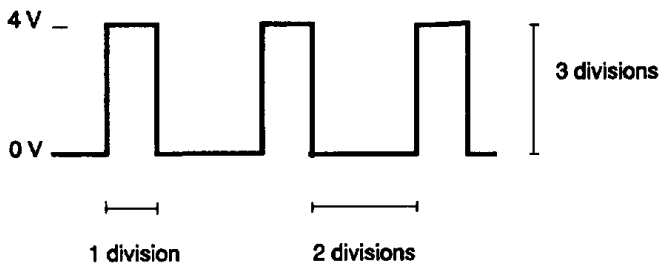

Fig. 4. A square wave to be realized.

find a correct sequence of adjusting the knobs and switches. Assignment 4. Confrontation between Real Signal and Oscilloscope Picture: The assignment reads: "Realize the signal in Fig. 4 and have it checked by the teaching assistant."

Note. Usually the students get the picture required on the oscilloscope screen. However, the divisions are correct but the amplitude and/or the period are not. The signal generator has no adjustment stops at $4 \mathrm{~V}$ amplitude, $0.4 \mathrm{~ms}$ period or a symmetry of 1 to 3 . This means that first the oscilloscope has to be used as a measuring apparatus (calibrated stops) while the continuous adjustment of the signal generator is used to get $4 \mathrm{~V}$ and $0.4 \mathrm{~ms}$. Second, the oscilloscope has to be used in continuous adjustment to get the divisions right while the signal generator adjustment should not be changed any more in this phase. Both the functions of the apparatus and the difference between the electrical signal and the picture on the screen are thus stressed.

The distinction between the different types of tasks, led to the design of new tasks like the examples given. The order of the tasks now becomes important.

\subsection{Tasks in Old and New Situations}

The laboratory course was changed in the academic year 1986-87. Before 1986-87 no clear distinction between preparative and comparative measurement assignments was made, nor were any open-ended tasks or confrontations present. From 1986-87 on a clear distinction has been introduced, and special tasks of all types were constructed and introduced. Of course students do not have to carry out the different types of tasks in the order presented in Section 2.1 but starting with the easier tasks.

During the first session they do an entrance test and receive remedial measurement assignments if necessary. After that they start with homework, either at home or in the next laboratory session. In the laboratory they fulfill introductory, preparative, comparative and open-ended assignments. Thus they learn to investigate but at the same time the students have to learn the content of the course, in its three parts. So in each part of the course there is an opportunity to train investigation. In the first part students have to get the idea, in the second part they practice it and apply it in the third part. Fitting results of measurements and calculations in a systematic way, even when not explicitly asked, is the intended final level of the course.

Preparative assignments are exercises in measurement procedures and calculations, that are not repeated in exactly 


\begin{tabular}{|c|c|c|c|c|c|c|c|c|c|}
\hline open ended & - & - & 1 & - & 1 & - & - & - & - \\
\hline comparing & - & 2 & - & 2 & - & 1 & 2 & 1 & 1 \\
\hline preparative & 2 & 2 & - & 2 & 1 & - & - & 1 & 4 \\
\hline homework & - & 10 & 5 & 4 & 6 & - & 14 & 3 & 4 \\
\hline session & 1 & 2 & 3 & 4 & 5 & 6 & 7 & 8 & 9 \\
\hline part of course & \multicolumn{3}{|c|}{ introductory } & \multicolumn{3}{|c|}{ time domain } & \multicolumn{3}{|c|}{ frequency dom } \\
\hline activity & \multicolumn{3}{|c|}{ orientation } & \multicolumn{3}{|c|}{ practise } & \multicolumn{3}{|c|}{ application } \\
\hline
\end{tabular}

Fig. 5. Distribution of types of assignments over the sessions after 1986-87. Homework tasks intend to let students read and combine information. Preparative assignments introduce new concepts. new functions of apparatus, or a new method to determine a variable. In comparing assignments the students compare the results of two methods and/or the methods themselves, and improve their performance until they get fitting results. In open ended assignments variables to be determined have to be specified, and appropriate methods for determination have to be found.

the same way later on. This means that students always have to construct new measurement or calculation procedures from the procedural elements embedded in the preceding assignments. Students are assigned the same tasks (analysis and synthesis of procedures) in several ways and in different disguises. Mastery learning (exercising prescribed procedures until they are mastered) is not intended. Students have to develop a strategy to investigate. Thus comparative tasks and open-ended assignments are considered higher level tasks because they require reflection on executional (procedural) skills. Homework e.g. is considered a lower-level task.

The distribution of the assignments over the sessions is presented in Fig. 5. The number of homework tasks have increased (from 40 to a total of 46) and the number of other assignments have diminished (from a total of 36 to 23). The types of assignments have been distributed more systematically over the sessions. Low level first, high level later in each domain; systematics of investigation introduced first, practiced or applied in the later domains. The first two parts of the course each contain one open-ended assignment. The last part contains comparative measurement assignments, i.e. the final level aimed at. It is thought that exercises at a higher than the final level in the previous parts of the course lead to more students acquiring the final level. The open-ended experiments could only be quite small because of the limited amount of time. Nevertheless, in order to learn to fulfill all tasks in time special care is required for information and assistance.

\subsection{Information and Teaching Assistance}

Assistance provides guidance in two ways. During the lab hours teaching assistants (TAs) are available to answer questions and to give hints. Giving hints without demonstrating how to do it, requires some experience of assistants.

In the old situation assistance was hectic. Much information had to be given orally and required long explanations, problems with apparatus had to be solved, students had to be hurried to finish their labs. The experienced TAs informed the fresh TAs in a haphazard way, usually only to raise criteria for assessing the logbooks.
In the new situation care was taken to provide for complete, but general information, distributed over different sections of the lab guide. This information could be studied by the students and referred to by the assistants.

A new section of the lab guide is devoted to the problems of ground and nodes, another to a functional description of the apparatus, and a third to the influence of measurements on the signal to be measured (with respect to amplitude, phase, or form). Concepts and skills to be learned in the course are stated, and the method of investigation is presented and explained (cf. Appendix). Finally, why, what and how to write in the logbook, receives attention. The lab write-up thus serves the purpose of learning to use a real logbook as in engineering practice is done.

Also teaching assistants now get a general training in assistance at labs, in which training experienced assistants turn (part of) their experience over to younger ones. Assistants are trained to ask students questions about what they think themselves, to give short answers, or to refer to available information probably overlooked or not understood by the student.

Another task of the assistants is to assess the lab logbooks and give feedback on the work and the progress of the students. This is particularly necessary in connection with the new (explicit) requirement to work methodically, and to journalize not only the results, but also the method of investigation. In the past this feedback differed among TAs, now the headings of the method of investigation can be used and feedback is more systematic.

The general purpose of the course is to learn to investigate in a systematic way. An important aspect of this goal concerns handling of information. Students have to learn to discover by themselves what information they need, and how to gather that information.

In the past they asked the assistants everything, including questions like: "Is this the correct answer, sir?" Discussions among students did not lead to a solution of their problems.

In the new situation they have to learn in the first part of the course that they have to find information themselves. Therefore the statements of the assignments are separated 
from the information needed to fulfill the tasks and from the information about the systematics of investigation. Part of the information is in the theoretical lecture notes and part is in different sections of the lab guide. In the first part of the course reference to the necessary information is made, later on the students have to find the relevant information themselves.

The lab guide starts with a special introduction to the subject. This introduction is concise, complete and understandable for students before they study the subject matter. It can be considered as a true abstract advance organizer in the sense of Ausubel [7] because it organizes the prior knowledge of the students rather than giving a-not yet understandable - outline of the course, thus providing anchoring points for new knowledge. The way this introduction is written is described elsewhere [3].

The relations between the changes in the lab course and the results of this intervention could be established by the way the changes were introduced and educational evaluations of the course.

\section{EDUCATIONAL RESUlts}

Data about the course are available from 1982 on. The general data (students' data and final marks for all courses) are available in a large data base at the university. Data concerning the performance of the students was acquired more precisely in the academic year 1990-91 [8] in the following way. Students were asked to answer a questionnaire after each session, while assistants were asked to grade a questionnaire sheet along with each assessment of a logbook. Three faculties made separate observations of students' behavior in the classrooms.

In this section we present an analysis of the marks and performance of the students, comparing the data before the change (especially 1985-86) and afterwards (especially 1989-90 and 1990-91).

\subsection{Marks of the Students Before and After the Change}

Five marks have been collected for each student: the mark for the entrance test, three successive gradings of the logbooks and the final mark for the laboratory course.

A survey of the final marks of students in the laboratory course over 8 years is presented in Fig. 6. Before 1986-87 the mean passing rate was $48 \%$ (immediate results during the course), from that year on it rose to $79 \%$. These results pertained until 1993 in which year the curriculum was completely changed. Also the total passing rate (after additional tasks in the following trimester) increased from $61 \%$ to $83 \%$. Thus more students passed the course in less time. It should moreover be mentioned that the time students took to complete the course (including time for preparation and completing the logbook) did not increase, but diminished slightly. It might be thought that students unjustly pass our course in the new situation, being helped too much (dragged). To answer this question results of good students in this course were compared with their results in other courses. Correlations between the marks for the labs were rather low (about 0.25) compared with the correlation between the marks for theoretical courses (about 0.50 ). Correlations between labs and theory were also

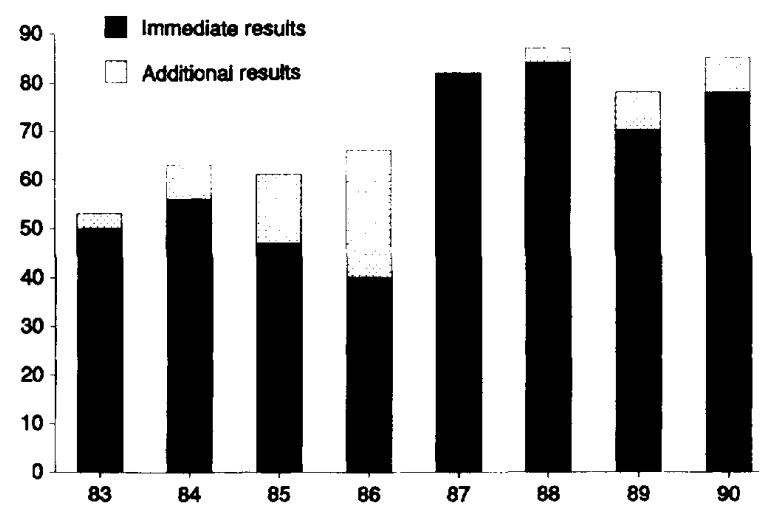

Fig. 6. Passing rates during eight years. Percentages of students of the entrance lists passing the lab course are shown. Immediate results refer to gradings obtained during the course. Additional results are obtained during the next trimester, holidays or next year.

low (about 0.25 ). Further analysis brought to light that after the change students expected to be good because of their earlier marks, scored higher and weak students scored lower in our course than before the change. Therefore the laboratory course in Network Analysis after the change provides a better discrimination between good and weak students than it did before the change.

The improved results of the course led to questions concerning the cause of this improvement.

\subsection{Performance of the Students After the Change}

We have checked a number of possibilities to explain the higher marks. The tasks were not easier thus the final level of performance has not changed. The total number of assignments has decreased somewhat, so mastery learning has not been the case. The time students spend on the course did not increase, so the effort of the students has not become greater. The average marks of the students for high school physics and mathematics were not higher before than after 1987. So the capabilities of the students have not changed. Neither have other courses been changed so much that an explanation for better performance in our course could be found. Remains an explanation based on teaching and learning in the course. Therefore we analyzed how the students learn in the new situation because of the new assignments and lab guide.

The data collected in 1990-91 were analyzed with regard to the performance and learning behavior of weak, moderate and good students. Good students in the course have a final mark of 8 or higher on a 1 to 10 scale, and amount to about $15 \%$ of the population. Moderate students score 6 or $7(65 \%)$, and weak students have unsatisfactory results, i.e. 5 or lower $(20 \%)$. We discuss the development in time during the course of results, general capabilities, and performance in assignments.

\subsection{Development of Performance}

The development of the results in time can be observed from the gradings of the logbooks and the marks for the entrance test (see Fig. 7). The results for the entrance test and the first 


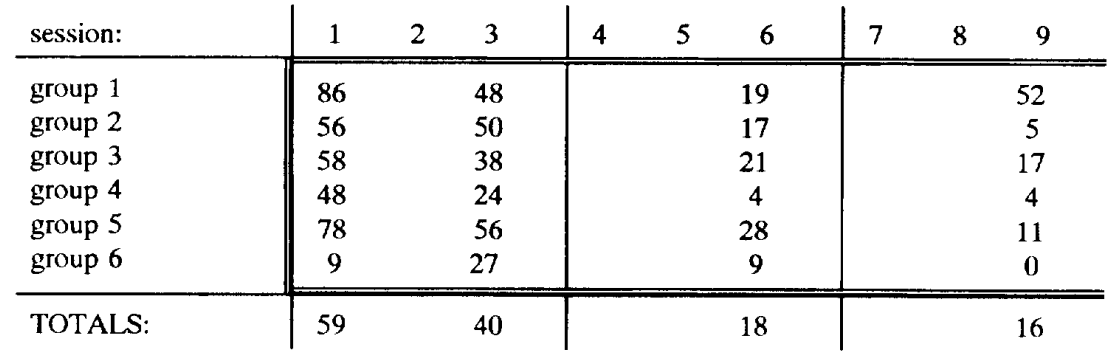

Fig. 7. Development of low performance during the course (new situation). The percentage of students performing below the level required is presented. Marks on the entrance test are obtained in session I, marks on the logbooks shortly after sessions 3, 6, and 9 .

logbook were rather bad $(59 \%$, respectively, $40 \%$ performing below level, another $13 \%$ dropped out). The results of the second and third logbooks, were much better. This means that most learning takes place between the first and the second part of the course, because of the feedback provided by the TAs in assessing the logbooks.

The results of the groups of about 25 students were generally good. Effects of the assistant grading the logbooks were unlikely because the assistants used explicit scoring criteria and each group was assessed by several assistants.

One group (group 1) showed a remarkable behavior. This group performed lowest on the entrance test, while only $50 \%$ of this group passed the course (just as in the past). The group had good marks for logbook 2, but bad marks for logbook 3 , indicating that these students did learn during two thirds of the course but that the load became too heavy during the last part of the course, the (new) frequency domain, although the assignments in part 3 were of a lower level.

The development of the performance of good, moderate and weak students also showed that weak students fail in the last part of the course. This does not explain why this effect is so strong in one group only. We suppose that the explanation can be found in the composition of the group. According to the results on the entrance test this was the weakest group of students. Probably the number of good students in this group was too low to generate the ideas necessary for sufficient progress.

Another remarkable effect is transfer of learning from the first to the last part of the course. Before the change many students complained that the concepts they had to use in the last part of the course (transfer function, Bode plot, polar diagram) had not yet been treated in the theoretical course. Therefore one of the strategies students had to learn in the new course, was to find by themselves the information they needed. This was included in the scheme for investigation and practiced in the course as already discussed. In the new situation some complaints still remain, but most students succeed in finding the necessary information in the last part of the course.

\subsection{Performance and Types of Assignments}

With respect to open-ended assignments it was found that all students improve their performance from the first to the second part of the course, the good students starting best and remaining best, the weak students improving least. Comparative assignments including measurements are at the core of the goals. Moderate students, starting at a lower level of performance than good students, end up at an equal level, while weak students, starting at a still lower level, stay at that level, and thus fail (cf. Fig. 8). It is often thought that good students need less time studying information than other students. Analysis of the data shows that good students spend most time of all students on answering homework assignments at home (see Fig. 9). Moreover, good and moderate students are better than weak students able to select the difficult and most relevant homework questions. Weak students spend most time on questions involving measurement schematics and practical subjects. The development of a number of general capabilities of the students has been monitored. These are the capability to reason, to study the laboratory manual, to study the lecture notes on Network Analysis, to understand the functions of the measurement apparatus, to compare different measuring methods with each other and to work methodically according to the scheme presented. It turned out that moderate students start at a lower level than good students, but improve their performance thereby approaching the good ones. The weak students stay roughly at the same low level they start at. In some cases, e.g. the capability to reason, weak students improve performance between the first and second part, but their performance drops in the third part.

\subsection{Behavior in Class}

It is remarkable how seldom the assistants are asked questions in the new situation. Interaction between all students is quite intensive however. Students often ask short questions to a student of another pair, or they go for a short look. Good students use least time for knowledge acquisition and most time for writing in the logbooks, for weak students the reverse is the case.

Good students ask the assistants better questions and need shorter answers than other students. The same applies to their contacts with other students. Weak students turn over pages in the manual more often than other students. It looks as if they do not know what and how to ask. They need longer explanations and fumble with the apparatus. Moderate students' behavior is in between. 
soore MEASUREMENT ASSIGNMENTS

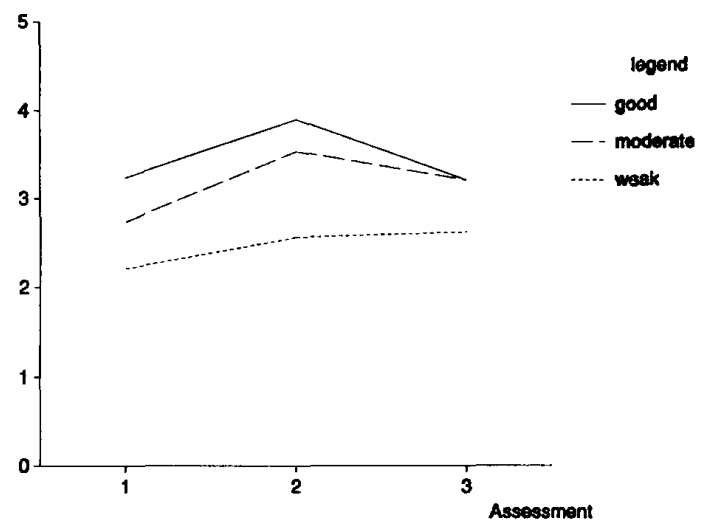

Fig. 8. Performance for measurement assignments (new situation). The relative scores of good, moderate and weak students on measurement assignments in the three logbooks are presented. These measurement assignments require inclusion of theory and analysis, and a comparison of results of measurements and calculations, even when not explicitly asked. The third part of the course is clearly more difficult (frequency domain).

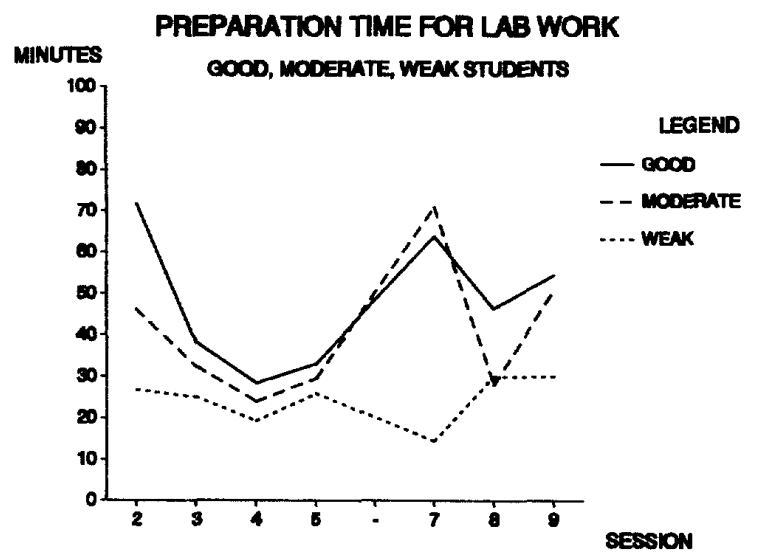

Fig. 9. Time spent by students for preparation of the lab tasks (new situation). The first and sixth session require no preparation. Good students spend most time on preparation and know when to spend it. Moderate students start spending less time but learn fast. Weak students start low and do not change their behavior.

Assistants are very busy the first session, especially during the entrance test and the remedial assignments. In later sessions they mostly spend their time grading the logbooks.

Students mostly find their own way in the lab. It can be said that the oral and partly insufficient instruction in the old situation has been successfully replaced by written instruction.

\section{CONCluSION AND DISCUSSION}

The conclusion is that a higher output is attained with the same goals. First, the course is more effective because more students attain the goal. Second, the course is more efficient because these goals are reached within a shorter time (within one trimester), while the effort of students and assistants is less than before. The question remains by what method and means these educational results are obtained.
In the past, during an assignment the students met too many difficulties at the same time making it difficult to fulfill their tasks, from the beginning on. In the new situation the difficulties are separated and stepwise overcome in the first part of the course. The difficulties in the last, unchanged part of the course can now be coped with by themselves. The students get a good grip on the concepts and techniques they need before they start, both at the beginning of the course and before the open ended assignments. They get support for systematically experimenting by means of the scheme for investigation. Thus they learn to think about the experimental set-up before or while working with it.

We conclude that the improved results are due to sufficient written information, and the type and order of the assignments designed. The information in the lab guide was much more than before, while the time spend by the students did not increase. Thus other aspects must be important too.

The teaching assistants were instructed-as in the past- to let the students themselves formulate the questions. In the past this instruction was not effective, now it is (for the groups in general). Likewise the interaction between the students in the past was not effective, and now it is. Also there is an indication that the groups should contain a minimum number of good students. This leads to the conclusion that well designed written material and assignments should be completed by an exchange of good ideas.

It appears that the teaching assistants do little work, and that their presence is superfluous. However, we prefer the conclusion that teaching assistants, by not showing initiative to help students, stimulate them to learn independently.

A good order of the assignments is difficult to find because quite a number of tasks lie at a high level. Here three types of learning tasks above a procedural level of knowledge are found: flexible use of procedures, comparative assignments, open-ended tasks. No procedures can be prescribed to carry out these tasks. At most heuristics can be given like the scheme for investigation. The students have to develop a strategy for constructing new procedures, for comparing results and procedures and for obtaining fitting results, and thus for investigation.

The nature and order of the tasks given to students depend generally on the experience and intuition of the staff responsible for a course. In our case of high requirements this turned out to be insufficient for designing a course. Therefore a scheme of double sequencing has been designed (cf. Section II and [9]). First, the assignments are arranged from a low level to a high level in each of the three parts of the course. Second, an introduction to the strategy of investigation is given in the first part, this strategy is practiced in the second part, while the strategy in the third part is applied to a new domain of the subject matter.

An educational measurement was carried out to investigate the learning process of the students. It turned out that weak students fail mostly in the last part only, showing that the course is selective with respect to transfer of strategy to a new domain. The impression that weak students do not get any help and may be discouraged, might be partly true. However, we do not know if they are slow-starters, or not suitable for electrical 
engineering, or just looking around to make another choice of department. Their number is small in any case (about 10\%). This number has to be compared to an overall total of about $40 \%$ of the students changing department in the first year. Remediation in the next trimester or year is still possible, moreover. And finally, our course should contribute to the selection of suitable students.
Happily, most of the time all students do learn, good, moderate and weak students alike, maybe a different amounts at a different pace.

Looking back we conclude that the course has changed from remedial teaching (tackling the shortcomings of the students in later trimesters) to preventive teaching (giving each student the possibility to learn).

\title{
APPENDIX
}

\author{
A SCHEME FOR INVESTIGATION
}

\section{$\underline{\text { PHASE }}$}

\section{ACTIVITY}

THE PROBLEM

formulate

analyze

INFORMATION

gathering

interpreting

HYPOTHESES

formulate

select

TEST EXPERIMENT

design

evaluate

CONCLUSIONS

draw

REPORTING
1. what is exactly asked?

2. try to focus and confine the problem

3. separate (partial) problems

4. represent the connections between the partial problems in a scheme

5. which variables play a role?

6. which relations contain these variables?

7. which relations contain quantities that relate to the variables?

8. what are the criteria for a satisfactory solution to the problem?

11. what information is needed in each phase/ activity? (about variables, relations, signals, networks, measurement procedures, etc.)

12. gather information from literature, lecture notes, lab guides, etc.

13. gather information by preliminary measurements.

14. analyze and interpret information, calculate signals and system behavior.

15. focus information onto the problem, select consequences. if an hypothesis (idea) turns out to be correct, it helps solving the problem.

21. hypotheses about signals or network models if useful relations are found

22 . hypotheses if relations need further investigation

23. select hypotheses that you want to test

24. consider all kinds of boundary conditions (available time, apparatus, etc.)

25. what are the criteria for a test experiment?

31. which variables can be adjusted in a measurable way, can be kept

constant, cannot be manipulated?

32. find other quantities that are related to these variables and that can be adjusted in a measurable way.

33. write down how these quantities can be measured

34. compare different measuring methods and make a choice

35 . consider the required accuracy and precision and available apparatus and time

36. write down a global plan of work

37. write down a step by step detailed measuring procedure (cookbook)

41. execute the procedure

42. analyze how precise and accurate your observations and results are

43. check any suppositions and limitations in connection with the criteria.

44. possible improvements of the investigation

51. draw conclusions about the hypotheses; formulate new ones if necessary.

52. check if the problem has been solved

53. formulate a final conclusion

61. write down all your thoughts, observations, dos and don'ts in your logbook

62. make a report of your investigation

\section{ACKNOWLEDGMENT}

The course on Network Analysis as well as the laboratory course are products of the staff of the research group "Theory of Information, Communication and Systems" of the Depart- ment of Electrical Engineering of the University of Twente, Enschede, The Netherlands. We would especially like to thank Prof. Ir. E. W. Gröneveld, Drs. R. W. Brink and Ir. L. P. J. Veelenturf for providing the basis for this paper. 


\section{REFERENCES}

[1] R. Cinquepalmi, C. Dell'Aquila, M. T. Fogli-Mu-Ciacca, V. Picciarelli, R. Stella, and G. Verrone, "The relationship between Piaget-type questionnaire scores and academic achievements of engineering freshmen," IEEE Trans. Educ., vol. E-28, no. 2, pp. 111-114, 1985.

[2] F. Reif and M. St. John, "Teaching physicists' thinking skills in the laboratory," Amer. J. Physics, vol. 47, no. 11, pp. 950-957, 1979.

[3] H. Vos, "Learning and transfer: The use of germ learning," Tijdschrift voor Onderwijsresearch, vol. 16, no. 5, pp. 261-278, 1991. (In Dutch, English transcript in preparation).

[4] L. K. J. Vandamme, "Electrical engineering in the New Netherlands educational system," Eur. J. Eng. Educ, vol. 15, pp. 45-90, 1990.

[5] F. F. G. de Bruin and H. Vos, "A basic course in network analysis. I: Content, results, instruction, IEEE Trans. Educ., vol. 38, no. 1, pp. XX, Feb. 1995.

[6] P. S. Katz and T. E. Warner, "Writing as a tool for learning," IEEE Trans. Educ., vol. 31, no. 3, pp. 214-216, 1988.

[7] D. P. Ausubel, J. D. Novak, and H. Hanesian, Educational Psychology: A Cognitive View. New York: Holt, 1978

[8] J. A. Oosterhuis-Geers, H.E.P. Tattje, and H. Vos, "Germ of learning applied to a laboratory course in network analysis: Learning and teaching," Doc. 91-85, Onderwijskundig Centrum/Faculteit der Elektrotechniek, Twente University, Enschede, The Netherlands (in Dutch)
[9] H. Vos, "Two levels of sequencing in germ learning: Course and task levels," in European Conf. Educ. Res., Book of Summaries, Tj. Plomp, J M. Pieters, and A. Feteris, Eds. Enschede: University of Twente, Dept. of Education, 1992, vol. 1, pp. 228-321.

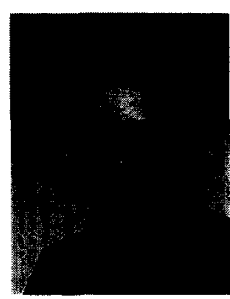

H.E.P. Tattje studied electrical engineering at the Polytechnical School of Utrecht.

In 1966 he joined the University of Twente as a Member of the Scientific Staff of the Department of Electrical Engineering in the Information, Communication and Systems Theory Group. Recently, he joined the Tele-Informatics and Open Systems Group. His main interests are speech and image coding and communication systems,

Dr. Tattje is a Member of the Dutch Electronics and Radio Engineering Society (NERG).

H. Vos, for biography and photograph, please see p. 6 of this issue 